Rabaska

Revue d'ethnologie de l'Amérique française

\title{
Centre canadien de recherche sur les francophonies en milieu minoritaire (Université de Régina)
}

\section{Sophie Bouffard}

Volume 9, 2011

URI : https://id.erudit.org/iderudit/1005955ar

DOI : https://doi.org/10.7202/1005955ar

Aller au sommaire du numéro

\section{Éditeur(s)}

Société québécoise d'ethnologie

ISSN

1703-7433 (imprimé)

1916-7350 (numérique)

Découvrir la revue

Citer ce document

Bouffard, S. (2011). Centre canadien de recherche sur les francophonies en milieu minoritaire (Université de Régina). Rabaska, 9, 386-388.

https://doi.org/10.7202/1005955ar d'utilisation que vous pouvez consulter en ligne. 
consultatif pour les subventions destinées au patrimoine, nous avons pu faire le traitement du fonds Le 100 Nons. Ce fonds documente l'évolution du 100 Nons, depuis sa création en 1967, et son importance pour la musique franco-manitobaine. Une collaboration avec le Collège universitaire de Saint-Boniface dans le cadre de l'ARUC a permis d'assurer, grâce aux services de Robert Nicolas, une partie du travail de tri de la collection de photographies de La Liberté. Robert Nicolas continuera son travail grâce à la subvention du Programme de subventions destinées au patrimoine.

Pour l'avenir, assurer un financement stable et durable du Centre du patrimoine est primordial. Maintenir les acquis, assurer un meilleur service à la clientèle et améliorer la préservation des archives et leur accès vont en dépendre. Cet objectif est maintenant celui qui exigera une attention particulière dans les années à venir.

Michel Lagacé, Gilles Lesage et Julie Reid

Centre canadien de recherche sur les francophonies en milieu minoritaire Institut français

Université de Régina

3737 Wascana Parkway

Régina (Saskatchewan) S4S 0A2
Téléphone : (306) 337-3273

Télécopieur : (306) 585-5183

Courriel : crfm.if@uregina.ca

Le Centre canadien de recherche sur les francophonies en milieu minoritaire (CRFM) de l'Institut français est le seul centre de recherche au Canada qui situe ses programmes au carrefour de la recherche universitaire, des enjeux auxquels fait face la minorité et du vécu de la communauté francophone. Fondé en 2005, le CRFM dirige, de façon multidisciplinaire et interinstitutionnelle, un programme de recherche novateur qui porte en priorité sur l'expérience de la communauté fransaskoise, tout en s'ouvrant aussi à celle d'autres populations francophones canadiennes et internationales. Le CRFM favorise également des recherches qui permettent de comparer cette expérience francophone à celle d'autres groupes ethnoculturels qui cherchent à s'affirmer en milieu minoritaire. Dominique Sarny en est le directeur depuis septembre 2010 et Sophie Bouffard, la coordonnatrice.

\section{Conférence de la Fondation Trudeau}

Le 31 mars 2011, pour souligner les 100 ans d'excellence en éducation à l'Université de Régina, l'Institut français accueillait, pour la première fois en Saskatchewan, une conférence dans le cadre de la prestigieuse série de conférences de la Fondation Trudeau. Les conférences Trudeau sont une série annuelle de présentations publiques permettant aux lauréats Trudeau de faire connaître les éléments clés de leur recherche. Monsieur Simon Harel, lauréat du Prix Trudeau 2009 et professeur au Département d'études littéraires de l'Université du Québec à Montréal, a présenté une conférence intitulée « De la migration à l'itinérance : le récit de soi et la mobilité contemporaine ». Spécialiste des questions qui touchent l'identité individuelle et l'intégration sociale, surtout chez les minorités, sa conférence abordait la capacité de se mouvoir entre 
des domaines culturels et, en particulier, la façon dont l'individu, tel l'immigrant et l'exilé, réagit à son environnement social.

\section{Conférences de l'Institut}

Pour la sixième année consécutive, le CRFM a présenté une série de conférences scientifiques grand public intitulée Les Conférences de l'Institut. Ces présentations portent sur les enjeux actuels spécifiques aux francophonies en milieu minoritaire. En 2010-2011, les conférences suivantes ont eu lieu à l'Institut :

- «Étude sur les déterminants d'accès et d'utilisation des services en français dans les institutions de longue durée en Saskatchewan », par Jean-Marie NkongoloBakenda, Université de Régina, 14 octobre 2010 ;

- «Les utopies francophones de l'Ouest canadien », par Colin Coates, Chaire du Canada sur les paysages culturels canadiens, Collège universitaire Glendon, York University, 25 novembre 2010.

\section{Forum : le postsecondaire en français en milieu minoritaire}

Le 17 mars 2011, dans le cadre du projet « Développer la recherche et assurer le transfert des connaissances sur la jeunesse dans la francophonie canadienne », dirigé par Annie Pilote, Université Laval, et mené en partenariat avec l'Observatoire Jeunes et Société, le CRFM de l'Institut français a organisé un forum spécial sur les études postsecondaires en milieu francophone minoritaire. Le forum a débuté par un exposé général présenté par $\mathrm{M}^{\mathrm{me}}$ Pilote où elle abordait quelques enjeux ayant trait aux études postsecondaires en milieu francophone minoritaire : accès, migration, identité, etc. Cette présentation a été suivie d'une discussion à laquelle ont participé :

- Carla Ardell, Léa Beaulieu-Prpick, Maria Ferré et Justin Nenson, étudiants ;

- Paul-Émile L'Heureux, député de l'Assemblée communautaire fransaskoise (ACF) responsable du dossier postsecondaire ;

- Dominique Sarny, directeur du CRFM.

\section{Actes du colloque sur la cause Caron}

Le CRFM prépare les actes du colloque bilingue intitulé « Le Statut du français dans l'Ouest canadien : la cause Caron » qui a eu lieu les 19 et 20 février 2010 à l'Institut français. Organisé en partenariat avec l'Association des juristes d'expression française de la Saskatchewan (AJEFS), cet événement d'envergure nationale a réussi à sensibiliser plus de 200 participants provenant de tout le pays à l'importance de la cause Caron et à ses répercussions possibles sur l'avenir de la francophonie dans l'Ouest canadien. Cette publication devrait paraître à la fin de l'année 2011.

\section{Terroir et développement rural}

Au cours de l'année 2010-2011, le CRFM a activement travaillé à la poursuite du programme de recherche « Terroir et développement rural », une initiative conjointe de l'Institut français et de l'Assemblée communautaire fransaskoise (ACF). Ce projet, qui vise à appuyer un processus de développement identitaire et communautaire via l'élaboration d'un concept de terroir adapté aux réalités des communautés en situation minoritaire, a pour objectif ultime d'entraîner une transformation des pratiques de 
développement rural dans la province. Démarche originale, cette initiative universitécommunauté comporte deux volets connexes : une composante recherche dirigée par le CRFM et un volet communautaire dirigé par l'ACF.

- Diagnostic global - Ludivine Tatieu-Bilhère s'est jointe à l'équipe de l'Institut français de septembre 2010 à avril 2011. Agronome de formation et forte de son expérience dans le domaine de la revitalisation rurale par le concept du terroir, notamment en France et en Espagne, elle a dressé un diagnostic global du potentiel de la région de Batoche (région pilote) et formulé des recommandations stratégiques quant aux prochaines étapes à franchir dans ce programme de développement rural. - Rencontre rurale "Terroir, culture et identité »- L'Assemblée communautaire fransaskoise $\left(\mathrm{A}_{\mathrm{CF}}\right)$ et l'Institut français ont organisé une rencontre rurale dans la région de Batoche du 25 au 29 mars 2011. Cette rencontre, qui visait à explorer de nouvelles alternatives en matière de revitalisation rurale, a aussi permis d'accueillir une délégation de représentants de la région de Charlevoix (Québec), chefs de file dans le développement du terroir en Amérique du Nord. Cet événement s'inscrit dans un projet interprovincial spécial de trois ans permettant de favoriser le partage de connaissances et de savoir-faire entre la région de Batoche et celle de Charlevoix.

\section{Affiliation importante}

L’Université de Régina est désormais membre de l'Agence universitaire de la francophonie (AUF), le premier réseau universitaire francophone mondial. La demande d'affiliation de l'université, proposée et préparée par le CRFM de l'Institut français, découle d'un engagement de longue date envers l'éducation et la recherche francophones à l'Université de Régina. Puisque l'Institut français représente l'Université de Régina auprès du public dans des contextes francophones, c'est lui qui représente l'université au sein de l'Auf et qui fait connaître les initiatives de celle-ci sur le campus.

\section{Partenariats et collaborations}

Au cours de l'année 2010-2011, le CRFM a également participé aux projets suivants : - Production d'articles sur le patrimoine culturel français de l'Ouest et du Nord canadiens pour l'Encyclopédie du patrimoine culturel de l'Amérique française ;

- Partenaire du colloque bilingue international SONIMAGE : The Legacies of Jean-Luc Godard présenté à l'Université de Régina du 16 au 18 septembre 2010 ;

- Collaboration à la Coalition pour la promotion de la langue française et la culture francophone en Saskatchewan ;

- Partenaire de l'Observatoire Jeunes et Société dans le cadre du projet « Développer la recherche et assurer le transfert des connaissances sur la jeunesse dans la francophonie canadienne » dirigé par Annie Pilote (Université Laval).

SOPHIE BOUFFARD 